\title{
How to microwave your tea (but using a kettle is better)
}

When a kettle of water is heated on a hob, convection currents within the fluid ensure that the heat spreads evenly throughout the liquid and the water reaches a uniform temperature.
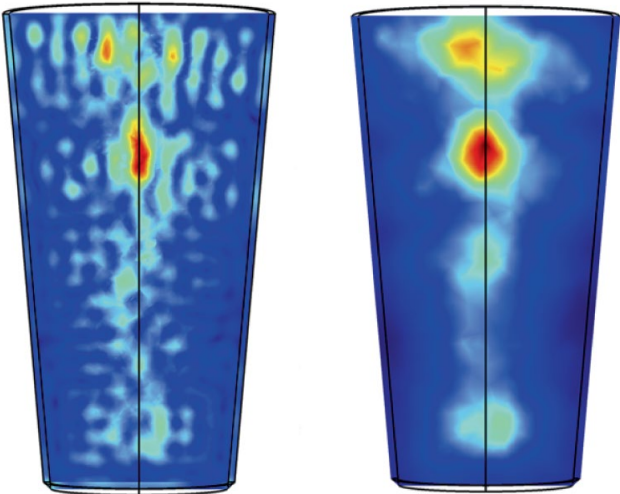

Credit: Image adapted from original article, CC BY 4.0 (https:// creativecommons.org/licenses/by/4.0/).
Yet, if you microwave a glass of water, the water at the top ends up much hotter than the bottom. Writing in AIP Advances, Peiyang Zhao and colleagues report the unusual convection process that leads to this temperature gradient and propose a solution.

In a microwave oven, the electric field distribution through the liquid corresponds closely to the temperature distribution of the liquid. This leads to local hot spots forming throughout the water (pictured), which contribute to a convection current. However, microwaves also directly heat the top of the water - which is not shielded by the glass as the sides and bottom are - and so hot water begins to gather at the top of the glass.
To improve the temperature distribution, Zhao and colleagues modified the top of the glass by coating it with a thin layer of silver. The metallic coating acts as a circular waveguide that attenuates the microwaves so that the electric field at the top of the glass is weakened. This enables the convection current caused by the local hot spots to dominate, leading to a more uniform temperature distribution. Silver-coating glasses at home may not be feasible, but using a metal lid showed a similar effect.

Ankita Anirban

ORIGINAL ARTICLE Zhao, P. et al. Multiphysics analysis for unusual heat convection in microwave heating liquid. AIP Adv. 10, 085201 (2020) 